\title{
Éditorial
}

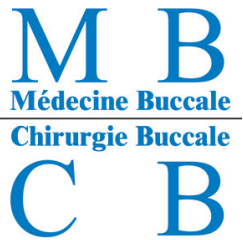

\section{Vous avez sans doute quelque chose à écrire ?}

\author{
Jacky Samson*
}

«L'excellence est un art que l'on n'atteint que par l'exercice constant » Aristote

Après la reconnaissance définitive de la Chirurgie orale comme spécialité malgré l'action ultime de quelques sycophantes, la Société française de Chirurgie orale souhaite maintenant obtenir le référencement de la revue Médecine Buccale Chirurgie Buccale (MBCB) qu'elle a créée ily a tout juste 20 ans. La parution initiale a été quelque peu erratique mais la revue $M B C B$ a néanmoins trouvé rapidement une stabilité tout en poursuivant la valorisation de son contenu. Cette évolution, d'abord le fait de quelques uns, n'a pu se faire qu'avec le concours du plus grand nombre, ou presque. Tous ceux qui sont spécialistes en Chirurgie orale, qui pratiquent ou qui s'intéressent à cette spécialité devraient être convaincus qu'ils sont liés par un contrat synallagmatique avec la revue MBCB. À terme, cette revue doit devenir un spicilège (les autres articles étant publiés en ligne) mais ils nous restent bien du chemin avant de parvenir à ce stade.

Afin de respecter les exigeances minimales pour demander le référencement, vous avez pu constater que la rubrique « Pour les auteurs » sur le site de la revue MBCB (mbcb-journal.org) a été totalement refondue, que la soumission se fait maintenant en ligne et que tous les articles sont transmis à deux reviewers qui les évaluent et qui s'efforcent d'émettre des remarques pertinentes sur le travail qui leur a été présenté, toujours dans le but d'améliorer la qualité. Il ne faut pas considérer le reviewer comme un contempteur car tout ceci se fait et doit se faire dans un esprit positif et confraternel. Après quelques mois de pratique, on a pu constater que peu d'auteurs se conforment aux « Conseils aux auteurs » - en particulier, pour la présentation des références bibliographiques -, que les auteurs ne répondent pas toujours aux sollicitations des reviewers... que certains reviewers s'investissent peu, que d'autres n'ont pas toujours lu les «Conseils aux auteurs» ... La rédaction d'un article médical scientifique répond à des régles dénuées de toute affêterie, une sorte de protocole opératoire rigoureux, qui sont clairement définies [1]. On ne peut s'y soustraire sous peine de voir son travail rejeté ou déconsidéré. Pour favoriser la diffusion et l'application de ces régles, la Société française de Chirugie orale a programmé un séminaire sur l'« Aide à la rédaction et à la soumission en ligne d'un article médical » le jeudi matin 22 mai 2014, à Reims. Ce séminaire auxquels vous êtes tous conviés (aussi bien les auteurs, reviewers de demain, que les reviewers d'aujourd'hui, auteurs d'hier et de demain), se fera sur un mode interactif et sera animé par Sylvain Catros, Jean-Christophe Fricain, Philippe Lesclous et Jacky Samson, qui présenteront successivement chacun deux ou trois thèmes pour ouvrir les débats. Il est bien évident qu'en une matinée, nous n'aurons pas le loisir d'aborder en détails tous les aspects relatifs à la rédaction d'un article médical. Selon l'accueil réservé à ce séminaire, on pourra envisager de poursuivre cette démarche et d'aborder des sujets connexes comme la différence entre auteurs et contributeurs, la définition du plagiat...

La plupart des revues ne définissent pas clairement le rôle d'un auteur et elles n'exigent pas de précisions sur la contribution de chaque auteur, sans doute parce que le comité de rédaction d'une revue n'a aucune légitimité pour récuser un auteur. Ainsi, on ne sait pas toujours, par exemple, ce que représente réellement le premier auteur, le dernier auteur, l'auteur correspondant, l'ordre des auteurs. Cependant, on voit se dessiner une tendance faisant référence à l'intégrité scientifique : elle préconise que le rôle de chacun soit parfaitement défini et connu. Sur ce sujet, l'ICMJE paraît un peu en retrait [2]. Il considère que le terme « auteur désigne généralement toute personne ayant fait une contribution intellectuelle substantielle à une étude publiée... ». Et il précise qu'il faut remplir trois conditions car « l'attribution de la qualité d'auteur doit reposer sur :

1) des contributions substantielles à la conception et la méthode, à l'acquisition de données ou à l'analyse et l'interprétation des données ;

2) la rédaction de l'article ou sa révision critique impliquant une contribution importante au contenu intellectuel ; et

3) l'approbation finale de la version à publier. »

\footnotetext{
*Correspondance : jacky.samson74@gmail.com
} 
Pour certains [3], cette définition est bien trop floue. Toutefois, si elle était scrupuleusement respectée, les « medical writters » seraient toujours bien identifiés et on verrait disparaitre les « guest authors », les " honorary authors » et les « ghost authors », termes qui varient en fonction du service rendu mais qui désignent toujours un auteur fantôme, voire fantoche, n'ayant bien souvent même pas lu l'article et n'ayant pas toujours été informé au préalable que son nom figure parmi les auteurs.

Devant l'importance donnée aux publications, très bien résumée dans la célèbre expression «publish or perish », les auteurs doivent constamment chercher à augmenter le nombre de leurs publications. Pour atteindre cet objectif, en dehors d'une démarche parfaitement intègre, ils peuvent avoir recours à trois subterfuges élémentaires : «publier des articles de peu d'intérêt, multiplier le nombre des auteurs de chaque article, ou diviser un article en plusieurs autres sur des sujets limités » [1]. Pour chacune de ces démarches, on prend toujours un peu plus de liberté avec l'intégrité scientifique. Certains n'hésitent pas à faire un pas de plus et à recourir au plagiat, à la falsification, voire à la fabrication de résultats. En 2013, le Comité de rédaction a eu à traiter deux cas de plagiat, l'un classique et bénin (« plagiaire hédoniste »), l'autre atypique et irrémissible (« plagiaire narcissique » ou « plagiaire autistique » car nous n'avons pas terminé l'étude du cas, cf. ci-dessous) [4]. Dans le premier cas, le passage incriminé a été retiré et l'article initialement plagié cité dans les références. Dans le deuxième cas, le plagiaire s'est manifesté de façon inhabituel : il nous a adressé un mail comminatoire demandant le retrait d'un article qui venait de paraître en ligne pour plagiat de plusieurs passages de sa thèse. Même si l'auteur principal de l'article incriminé ne pouvait guère être suspecté d'un tel forfait, nous l'avons informé et nous avons essayé ensemble de comprendre sur quoi pouvait reposer une telle accusation. Après élimination du bruit de fond (accusation portant sur l'emprunt de quelques idées), l'accusation ne reposait plus en fait que sur une phrase. Or, cette phrase de quelques mots provenait du texte d'une recommandation émise par l'AFSSAPS ! Comme les gens malhonnêtes sont persuadés que tout le monde fonctionne comme eux, nous avons demandé à l'exaccusé (qui venait de faire une large revue de la littérature sur le sujet pour rédiger son article) s'il pouvait lire la thèse de l'accusateur pour rechercher d'éventuels passages plagiés. Avec une simple lecture, il a identifié plusieurs passages copiés-collés, « empruntés » à l'AFSSAPS, à un site internet et à deux articles que nous avions publié quelques années plus tôt. Le diagnostic étant confirmé, nous n'avons pas jugé bon de procéder à d'autres investigations. Pour terminer le traitement de ce cas, un rapport subséquent a été adressé aux autorités universitaires tutélaires du plagiaire qui, de plus, n'a pas jugé bon de s'excuser pour cette dénonciation calomnieuse et n'a manifesté aucune résipiscence pour le plagiat.

Vous avez sans aucun doute quelque chose à écrire d'original et, en rédigeant votre article, ayez toujours à l'esprit que l'image de la revue MBCB constitue le reflet du niveau scientifique de notre spécialité.

« N'importe quelle idée semble personnelle dès qu'on ne se rappelle plus à qui on l'a empruntée» Jules Renard

Conflits d'intérêt : aucun

\section{Références}

1. Maisonneuve $\mathrm{H}$, Lorette $\mathrm{G}$, Maruani $A$, Huguier $M$. La rédaction médicale, $5^{\mathrm{e}}$ ed. Doin Editeurs, Rueil-Malmaison, 2010.

2. Recommandations de l'International Committee of Journal Medical Editors (ICMJE). http://www.icmje.org/french.pdf.

3. Académies suisses des sciences. Qualité d'auteur des publications scientifiques. Analyse et recommandations, 30 mai 2013. www.academies-suisses.ch / publications / directives et recommandations.

4. Maurel-Indart H. Petite enquête sur le plagiaire sans scrupule. Editions Léo Scheer, Paris, 2013. 